\title{
THE KODAIRA DIMENSION OF THE MODULI SPACE OF CURVES OF GENUS 15
}

\author{
MEI-CHU CHANG \& ZIV RAN
}

\section{Introduction}

The purpose of this paper. is to prove that the moduli space $\mathscr{M}_{15}$ of curves of genus 15 has Kodaira dimension $\kappa=-\infty$, i.e. that $H^{0}\left(\mathscr{M}_{15}, n K_{\mathscr{M}_{15}}\right)=0$ for all $n>0$.

We recall briefly the current state of affairs regarding the structure of $\mathscr{M}_{g}$ : $\mathscr{M}_{g}$ is unirational (in particular, has $\kappa=-\infty$ ) for $g \leqslant 13$ ([2], [4], [5], [13]), has $\kappa \geqslant 1$ for all $g \geqslant 23$, and is of general type for $g \geqslant 24$ ([6], [8], [9]).

Our proof is based on an analysis of a particular divisor $D \subset \overline{\mathscr{M}}_{15}$, namely

$$
D=\text { some component of the locus of curves carrying a } g_{14}^{3} \text {. }
$$

We show that $D$ is unirational. Moreover, for some rational curve $F \subset D$ which is a member of a family of rational curves "filling up" $D$, we show that the intersection numbers

$$
D \cdot F>0, \text { while } F \cdot K_{\mathscr{M}_{15}}<0 .
$$

As is easily seen, this implies $\kappa\left(\mathscr{M}_{15}\right)=-\infty$.

Our analysis of $D$ is based on a correspondence

$$
\mathscr{N} \leftarrow \rightarrow \mathscr{H} \longrightarrow \rightarrow \mathscr{M}_{15}
$$

where $\mathscr{H}$ is the closure of some component of the Hilbert scheme of nonsingular curves of genus 15 and degree 14 in $\mathbf{P}^{3}$, and $\mathscr{H} \rightarrow \rightarrow \mathscr{M}_{15}$ is the natural rational map. On the other hand, essentially, $\mathscr{N}=$ space of $4 \times 9$ matrices

$$
A=(\underbrace{}_{8} \begin{array}{ll}
L & Q
\end{array}),
$$

Received November 18, 1985. The first author was partially supported by a Rackham fellowship and the second was partially supported by the National Science Foundation. 
where $L$ signifies linear forms and $Q$ signifies quadratic forms on $\mathbf{P}^{3}$. The map $\mathscr{N} \rightarrow \mathscr{H}$ is given by $A \mapsto$ dependency locus of $B$, where $B$ is the essentially unique $9 \times 4$ matrix

$$
\left.\left(\begin{array}{c}
Q^{\prime} \\
L^{\prime}
\end{array}\right)\right\} \begin{aligned}
& 8 \\
& 8
\end{aligned}
$$

such that $A B=0$. The inverse map $\mathscr{H} \rightarrow \rightarrow \mathscr{N}$ is given by a Serre construction plus a monad construction. This correspondence is analogous to the one used in our proof [4], [5] of the unirationality of $\mathscr{M}_{g}$ for $g \leqslant 13$. But the main departure from [5], to which the bulk of the paper is devoted, is a partial study of this correspondence in codimension 1-rather than just at generic points. In particular, the locus $V_{0}$ of nonmaximal rank curves in $\mathscr{H}$-over which the correspondence $\mathscr{N} \leftarrow \rightarrow \mathscr{H}$ blows up-plays an essential role in the existence of $F$.

Roughly speaking, our proof goes as follows. We start with a rather common and explicit sort of pencil $F_{0}$ of curves in $\mathscr{H}$, which itself, like-it would seem -any pencil one can construct "explicitly," could not possibly have properties (*) above. We then lift $F_{0}$ to a pencil of matrices in $\mathscr{N}$. Since $\mathscr{N}$ is just a linear space, the latter pencil can be moved around freely, and we then map it over to $\mathscr{M}_{15}$, which yields a rational curve $F$ as above. Using formulas of Eisenbud-Harris [6] and Mumford [12] for the classes of $D$ and $K_{\mathscr{M}_{15}}$ in $\operatorname{Pic}\left(\mathscr{M}_{15}\right)$, respectively, we establish (*) above.

We emphasize that $F$ is not the image of $F_{0}$ : this has to do with the fact that $F_{0}$ meets $V_{0}$, over which the correspondence $\mathscr{N} \leftarrow \rightarrow \mathscr{H}$ blows up; it is the latter fact that, ultimately, allows $F$ to be constructed.

Note that, since our methods apply only to curves $Y$ in $\mathbf{P}^{3}$ with $H^{1}\left(\mathscr{I}_{Y}(5)\right)$ $=0$, it follows that genus 15 is the only genus $g \geqslant 14$ for which curves of this type fill up at least a divisor in $\mathscr{M}_{15}$.

We note finally that the curve $F$ above comes very "close" to making $\mathscr{M}_{15}$ uniruled, but does not quite make it; i.e. it cannot be (flatly) deformed out of $D$. This comes from the fact that $D$ crosses itself at some points of $F$, and while $D \cdot F \geqslant 0$, the "normal bundle to the branch" is negative on $F$. On the other hand, it is apparently conjectured in general that $\kappa=-\infty$ implies uniruledness. Thus $\mathscr{M}_{15}$ seems like a good test case for this conjecture.

Notations and conventions. In this paper we work over the complex numbers. A curve $Y$ is a connected, projective, Cohen-Macaulay, 1-dimensional scheme, usually with a given embedding in projective space. $\omega_{Y}$ denotes the dualizing sheaf, and $\mathscr{I}_{Y}$ the ideal sheaf of $Y$. If $Y$ is a locally complete intersection, $N_{Y}$ denotes the normal bundle, i.e. $\mathscr{H}_{o} m\left(\mathscr{I}_{Y}, \mathcal{O}_{Y}\right)$. If $H^{1}\left(N_{Y}\right)=0$, 
then the Hilbert scheme is smooth in a neighborhood of $Y . \mu_{0}$, or $\mu_{0}(Y)$, denotes the multiplication mapping $H^{0}\left(\omega_{Y}(-1)\right) \otimes H^{0}\left(\mathcal{O}_{Y}(1)\right) \rightarrow H^{0}\left(\omega_{Y}\right)$. For a discussion of $\mu_{0}$, see [1], [14]. $Y$ has maximal rank if the restriction mappings $H^{0}\left(\mathcal{O}_{\mathbf{P}^{n}}(k)\right) \rightarrow H^{0}\left(\mathcal{O}_{Y}(k)\right)$ have maximal rank for all $k . H_{d, g}$ denotes the closure of the Hilbert scheme of nonsingular curves of degree $d$, genus $g$ in $\mathbf{P}^{3}$.

\section{Relative Serre construction and relative monads}

We begin by stating a relative version of the Serre construction.

Proposition 1.1. Let $\mathscr{Y} \subset B \times \mathbf{P}^{3} \stackrel{\pi}{\rightarrow} B$ be a flat family of Cohen-Macaulay curves and let $V$ be a (locally split) subbundle of $\pi_{*}\left(\omega_{o / B}(A, b)\right)$, with $b \leqslant 3$, such that the map $\pi^{*} V \rightarrow \omega_{\mathscr{Y} / B}(A, b)$ is surjective. Here $\mathcal{O}(A, b)=\pi^{*} A \otimes$ $p^{*} \mathcal{O}(b)$, where $A$ is a line bundle on $B$ and $p: B \times \mathbf{P}^{3} \rightarrow \mathbf{P}^{3}$ is the projection. Then there exists canonically a vector bundle $\mathscr{E}$ on $B \times \mathbf{P}^{3}$ as an exact sequence

$$
0 \rightarrow\left(\pi^{*} V\right)^{\vee} \rightarrow \mathscr{E} \rightarrow \mathscr{I}_{\mathscr{Y}}\left(A^{\vee}, 4-b\right) \rightarrow 0
$$

with dual

$$
0 \rightarrow \mathcal{O}(A, b-4) \rightarrow \mathscr{E}^{\vee} \rightarrow \pi^{*} V \rightarrow \omega_{\mathscr{Y} / B}(A, b) \rightarrow 0 .
$$

Proof. This is proved by relativizing the proof of the usual Serre construction [11], so we just sketch the additional argument. We have

$$
\omega_{\mathscr{Y} / B}(A, b)=\mathscr{E} x t^{2}\left(\mathcal{O}_{\mathscr{Y}}, \omega_{\mathbf{P}^{3} \times B / B}\right)(A, b)=\mathscr{E} x t^{2}\left(\mathcal{O}_{\mathscr{Y}}, \mathcal{O}(A, b-4)\right) .
$$

The map $\pi^{*} V \rightarrow \omega_{\mathscr{Y} / B}(A, b)$ yields a section of

$$
\begin{aligned}
\omega_{\mathscr{Y} / B}(A, b) \otimes \pi^{*} V^{\vee} & =\mathscr{E} x t^{2}\left(\mathcal{O}_{\mathscr{Y}}, \mathcal{O}(A, b-4) \otimes \pi^{*} V^{\vee}\right) \\
& =\mathscr{E} x t^{1}\left(\mathscr{I}_{\mathscr{y}}, \mathcal{O}(A, b-4) \otimes \pi^{*} V^{\vee}\right) .
\end{aligned}
$$

Since $b \leqslant 3$, we have $\pi_{*}\left(\mathscr{H}_{o m}\left(\mathscr{I}_{\text {oy }}, \mathcal{O}(A, b-4)\right) \otimes \pi^{*} V^{\vee}\right)=0$, so the Hochschild-Serre sequence yields

$$
\begin{aligned}
H^{0}\left(\mathscr{E} x t^{1}\left(\mathscr{I}_{\text {Oy }}, \mathcal{O}(A, b-4) \otimes \pi^{*} V^{\vee}\right)\right) & \simeq \operatorname{Ext}^{1}\left(\mathscr{I}_{\text {Oy }}, \mathcal{O}(A, b-4) \otimes \pi^{*} V^{\vee}\right) \\
& =\operatorname{Ext}^{1}\left(\mathscr{I}_{\mathscr{O}}\left(A^{\vee}, 4-b\right), \pi^{*} V^{\vee}\right) .
\end{aligned}
$$

This extension is $\mathscr{E}$, and it is now straightforward to check the assertion of the proposition.

Remark 1.1.1. If $b \geqslant 4, \mathscr{E}$ still exists but may not be canonical.

Next, we compute the Chern classes of $\mathscr{E}$, assuming for simplicity $B$ is a smooth curve. Denote by $H_{1}, H_{2} \in H^{2}\left(B \times \mathbf{P}^{3}, \mathbf{Z}\right)$ the class of (pt.) $\times \mathbf{P}^{3}$, 
$B \times$ (plane), respectively, $a=\operatorname{deg} A$. Note that $H^{4}\left(B \times \mathbf{P}^{3}, \mathbf{Z}\right)=\mathbf{Z} \cdot H_{1} H_{2} \oplus$ $\mathbf{Z} \cdot H_{2}^{2}$ with $H_{1} H_{2} \cdot H_{2}^{2}=1,\left(H_{1} H_{2}\right)^{2}=\left(H_{2}^{2}\right)^{2}=0$. For a cycle $Z \sim m H_{1} H_{2}$ $+n H_{2}^{2}$, note that $m$ is the degree of $p_{*} Z$, while $n$ is the degree of the restriction of $Z$ on the $\mathbf{P}^{3}$ factor.

Lemma 1.2. In the situation of Proposition 1.1, we have

$c_{1}(\mathscr{E})=(-a-c) H_{1}+(4-b) H_{2}, c_{2}(\mathscr{E})=(-c(4-b)+m) H_{1} H_{2}+n H_{2}^{2}$, where $a=\operatorname{deg} V, m=\operatorname{deg} p_{*}(\mathscr{Y})$, and $n$ is the degree of the generic fiber of $\mathscr{Y}$.

Proof. This follows easily from sequence (1.1), using the formula for twisting Chern classes [7, Example 3.2.2], plus Grothendieck's formula $c_{2}\left(\mathscr{I}_{\mathscr{O}}\right)$ $=-c_{2}\left(\mathcal{O}_{\mathscr{O}}\right)=[\mathscr{Y}][7$, Example 15.3.5].

Next, we want to construct a monad for $\mathscr{Y}$ and $\mathscr{E}$ as above, and we begin with the case of a single curve $Y$. In [5], we considered the case where $Y$ has its $\mu_{0}$ map injective; here we consider the case where $\mu_{0}$ is surjective. Then, as it turns out, the monad becomes "degenerate," i.e. a co-resolution.

Proposition 1.3. Let $Y \subset \mathbf{P}^{3}$ be a curve with the following properties:

(i) $Y$ is linearly normal;

(ii) $\mu_{0}(Y)$ is surjective;

(iii) $Y$ is not in a quadric;

(iv) $\mathcal{O}_{Y}(2)$ is nonspecial;

(v) the Brill-Noether number $\rho=4 d-3 g-12 \geqslant-3$.

Let $E(2)$ be the canonical extension

$$
0 \rightarrow H^{0}\left(\omega_{Y}(-1)\right)^{\vee} \otimes \mathcal{O} \rightarrow E(2) \rightarrow \mathscr{I}_{Y}(5) \rightarrow 0 .
$$

Then there is a canonical extension

$$
0 \rightarrow E \rightarrow B \rightarrow H^{1}\left(\mathscr{I}_{Y}(2)\right) \otimes \mathcal{O}(1) \rightarrow 0
$$

with $B$ (noncanonically) isomorphic to $(-\rho) \mathcal{O}(-1) \oplus \sigma \mathcal{O}$, where $\sigma=5 d-3 g-$ 17.

Proof (cf. [3]). Since $H^{1}\left(\mathscr{I}_{Y}(2)\right)=H^{1}(E(-1))=\operatorname{Ext}^{1}(\mathcal{O}(1), E)$, a canonical $B$ exists, so it is just a matter of identifying $B$. Sequence (1.3) and properties (i), (iii) yield $H^{1}(E(\leqslant-2))=0$, and $h^{1}(E(-1))=4$. Restricting (1.3) on a general line $L$ yields $H^{1}\left(E_{L}(\geqslant 1)\right)=0$. Hence as in [3], we see that $H^{1}(E(*))$ is generated by $H^{1}(E(-1))$ and $H^{1}(E)$. Moreover the dual sequence of (1.3) and properties (ii), (iv) yield $H^{1}\left(E^{\vee}(\leqslant-1)\right)=0$. Hence a minimal monad for $E$ is

$$
A \rightarrow B \rightarrow C
$$

where $A=\oplus \mathcal{O}\left(m_{i}\right), \quad m_{i} \geqslant 0, \quad B=\oplus \mathcal{O}\left(n_{i}\right), \quad$ and $\quad C=c \mathcal{O}(1) \oplus c^{\prime} \mathcal{O}, \quad c=$ $h^{1}\left(\mathscr{I}_{Y}(2)\right)=2 d-g-9$. By minimality, we have $m:=\max \left(m_{i}\right)<\max \left(n_{i}\right)$ $=: n$. Hence $H^{0}(B(-n)) \neq 0$ and by looking at the display of the monad we 
get $H^{0}(E(-n)) \neq 0$ which implies $n \leqslant 0$, hence $A=0$. A similar argument using $H^{0}\left(E^{\vee}(\leqslant-2)\right)=0$ yields $\min \left(n_{i}\right) \geqslant-1$. Since $c_{1}(B)=c_{1}(E)+c_{1}(C)$ $=-\rho$, we get $B=(-\rho) \mathcal{O}(-1) \oplus b \mathcal{O}$. By minimality, the map $B \rightarrow C$ induces the 0 -map $b \mathcal{O} \rightarrow c^{\prime} \mathcal{O}$. Since $-\rho \leqslant 3$, the map $-\rho \mathcal{O}(-1) \rightarrow c^{\prime} \mathcal{O}$ cannot be surjective unless $c^{\prime}=0$, so by comparing ranks the proposition is proved.

For a bundle $\mathscr{E}$ over $\mathbf{P}^{1} \times \mathbf{P}^{3}$ we can globalize the monad construction because of the following elementary result, whose proof we omit.

Lemma 1.4. If $B$ is a bundle over $\mathbf{P}^{1} \times \mathbf{P}^{3}$ such that for all $t \in \mathbf{P}^{1}$, $\left.B\right|_{t \times \mathbf{P}^{3}} \simeq \oplus k_{i} \mathcal{O}\left(n_{i}\right)$ for some strictly decreasing sequence of integers $n_{i}$ independent of $t$, then there is a canonical filtration $B_{1} \subset B_{2} \subset \cdots \subset B$ with associated quotients $\bigoplus_{j=1}^{k_{i}} \mathcal{O}\left(m_{i j}, n_{i}\right)$.

Proposition 1.5. Denote by $\mathscr{E}(0,2)$ the $\mathscr{E}$ of Proposition 1.1, and assume $b=-1$ and that for each $t, Y_{t}=\pi^{*}(t)$ satisfies the hypothesis of Proposition 1.3. Then there is a global monad

$$
0 \rightarrow \mathscr{E} \rightarrow B \rightarrow\left(R^{1} \pi_{*} \mathscr{I}_{\mathscr{Y}}(a, 2)\right) \otimes \mathcal{O}(0,1) \rightarrow 0
$$

with

$$
\begin{gathered}
0 \rightarrow \oplus \mathcal{O}\left(m_{i}^{\prime}, 0\right) \rightarrow B \rightarrow \bigoplus_{1}^{-\rho} \mathcal{O}\left(m_{i},-1\right) \rightarrow 0, \\
c_{1}(\mathscr{E})=\left(\sum m_{i}+\sum m_{i}^{\prime}-\operatorname{deg}\left(R^{1} \pi_{*} \mathscr{I}_{\mathscr{Y}}(a, 2)\right)\right) H_{1}+(2(g-d)+3) H_{2},
\end{gathered}
$$

and $c_{2}(\mathscr{E})$ may be computed. Moreover, if a generic $Y_{t}$ is not in a cubic, then

$$
\max \left(m_{i}^{\prime}\right) \leqslant \max \left\{m \mid \mathcal{O}(m) \subset R^{1} \pi_{*} \mathscr{I}_{\text {Oy }}(a, 2)\right\} .
$$

Proof. This follows from the above plus a Chern class computation. The last assertion comes from the fact that

$$
H^{0}(\mathscr{E})=H^{0}\left(\pi_{*} \mathscr{E}\right)=H^{0}\left(\pi_{*} \mathscr{I}_{\mathscr{Y}}(a, 3)\right)=0 .
$$

Example 1.6. Suppose in the situation above we have

$$
\begin{gathered}
a=1,[\mathscr{Y}]=H_{1} H_{2}+14 H_{2}^{2}, \quad \pi_{*} \omega_{\mathscr{Y} / \mathbf{P}^{1}}(0,-1)=\mathcal{O}(1) \oplus 3 \mathcal{O}, \\
R^{1} \pi_{*} \mathscr{I}_{Y}(0,2)=4 \mathcal{O} .
\end{gathered}
$$

Then we compute that

$$
c_{1}(\mathscr{E}(0,2))=4 H_{1}+5 H_{2}, \quad c_{2}(\mathscr{E}(0,2))=16 H_{1} H_{2}+14 H_{2}^{2} .
$$

Since $\Sigma_{1}^{8} m_{i}^{\prime}=8$ and all $m_{i}^{\prime} \leqslant 1$, we get $m_{i}^{\prime}=1, i=1, \cdots, 8$; also $m_{i}=0$. Hence the extension $0 \rightarrow \oplus \mathcal{O}(1,0) \rightarrow B \rightarrow \mathcal{O}(0,-1) \rightarrow 0$ splits, so the monad is

$$
0 \rightarrow \mathscr{E} \rightarrow \bigoplus_{1}^{8} \mathcal{O}(1,0) \oplus \mathcal{O}(0,-1) \rightarrow 4 \mathcal{O}(1,1) \rightarrow 0
$$




\section{Good pencils}

In this section we construct the good pencil of curves as described in the Introduction. As was said there, our strategy is to start with a rather simple pencil of curves of degree 14 , genus 15 in $\mathbf{P}^{3}$, which itself has no chance of being a specialization of a general pencil. However, we can still construct the corresponding pencil of bundles and matrices, and it turns out that the latter is the specialization of a general pencil of bundles and matrices, so by generalizing it and taking dependency locus we get a general pencil of curves (which, it turns out, fills out a surface of degree 16). What is happening here is that for the special pencil of bundles, the space of sections jumps (exactly three times, in fact).

We begin by considering curves of the type

$$
Y=Q \cup D,
$$

where $D$ is a curve of degree 10 , genus 3 , and $Q$ is a quartic in some plane $H$, containing the 10 points $D \cap H$. More precisely, we consider

$V=$ closure in the Hilbert scheme of the locus of curves $Y=Q \cup D$ as above such that $D \cap H$ imposes independent conditions on $\left|\mathcal{O}_{H}(4)\right|$.

Since the curves $D$ are parametrized by an irreducible variety $H_{10,3}$, it is clear that $V$ is irreducible. The fact that $V$ is nonempty follows from

Lemma 2.1. For a general $D \in H_{10,3}$, we have

(i) for a fixed plane $H, D \cap H$ consists of 10 generic points on $H$;

(ii) $H^{1}\left(N_{D}(-1)\right)=0$.

Proof. Given general points $P_{1}, \cdots, P_{10}$, take an elliptic quartic $E_{1}$ through $P_{1}, \cdots, P_{4}$, then another elliptic quartic $E_{2}$ through $P_{5}, \cdots, P_{8}$ plus some point on $E_{1}$, then a conic $C$ through $P_{q}, P_{10}$, one point of $E_{1}$, and one point of $E_{2}$. It is easy to see that $E_{1} \cup E_{2} \cup C$ is smoothable, and this establishes (i). For (ii), note the exact sequence

$$
\left.0 \rightarrow N_{D}(-1) \rightarrow N_{D} \rightarrow N_{D}\right|_{D \cap H} \rightarrow 0,
$$

where $N_{D}$ denotes the normal bundle. It is easily seen that $H^{1}\left(N_{D}\right)=0$, hence $H_{10,3}$ is smooth near $D$ with tangent space $H^{0}\left(N_{D}\right)$. Moreover, the induced map

$$
H^{0}\left(N_{D}\right) \stackrel{\phi}{\rightarrow} H^{0}\left(\left.N_{D}\right|_{D \cap H}\right)=\bigoplus_{P \in D \cap H} N_{D}(P)
$$

is just the differential of the map $H_{10,3} \stackrel{f}{\rightarrow} \operatorname{Sym}^{10}(H)$ given by $D \rightarrow D \cap H$. By (i), $f$ is dominant, hence $\phi$ is surjective for general $D$, therefore $H^{1}\left(N_{D}(-1)\right)=$ 0 , as claimed. 
Lemma 2.2. The curves in $V$ are smoothable.

Proof. Specialize $D$ to

$$
\bar{D}=E_{2} \cup L \cup C_{4} \cup C_{5} \cup C_{6},
$$

where $E_{2} \subset H_{2}$ is a plane cubic, $L$ is a line, $C_{i} \subset H_{i}$ are conics, and $E_{2}$ meets $C_{4}$ and $C_{5}$ in one point each, $L$ meets $C_{4}, C_{5}, C_{6}$ in one point each, and $C_{5}$ meets $C_{6}$ in one point. It is easy to check that for $\bar{D}$ general as above, $\bar{D} \cap H$ imposes independent conditions on $\left|\mathcal{O}_{H}(4)\right|$. Then specialize $Q$ to $\bar{Q}=C \cup C^{\prime}$, where $C$ is a conic meeting $E_{2}$ in two points and the rest of $\bar{D}$ in three points, and $C^{\prime}$ is a conic meeting $E_{2}$ in one point and the rest of $\bar{D}$ in four points. Then writing $\bar{Y}=\bar{Q} \cup \bar{D}$ as

$$
\left.\bar{Y}=\left(\left(\left(\left(C \cup E_{2}\right) \cup C^{\prime}\right) \cup L\right) \cup C_{4}\right) \cup C_{5}\right) \cup C_{6}
$$

and using the fact that $C \cup E_{2}$ is smoothable and has $H^{1}(N)=0$ (because nonspecial) plus Sernesi's result [14] that (smoothable, $\left.H^{1}(N)=0\right) \cup(4$ or 5-secant conic or 1-secant line) is smoothable and has $H^{1}(N)=0$, we see that $\bar{Y}$ is smoothable.

Lemma 2.3. If $Y=Q \cup D$ is in $V$ where $D$ is general and $Q$ is smooth at $D \cap H$, then $H^{1}\left(N_{Y}\right)=0$.

Proof. We have an exact sequence

$$
\left.\left.0 \rightarrow N_{Y}\right|_{D}(-1) \rightarrow N_{Y} \rightarrow N_{Y}\right|_{Q} \rightarrow 0 .
$$

Since $\left.N_{Y}\right|_{D}(-1)$ contains $N_{D}(-1)$ as a subsheaf of full rank and $H^{1}\left(N_{D}(-1)\right)=0$ by Lemma 2.1, we have $H^{1}\left(\left.N_{Y}\right|_{D}(-1)\right)=0$. To prove the lemma, it suffices to show that $H^{1}\left(\left.N_{Y}\right|_{Q}\right)=0$. Now the diagram

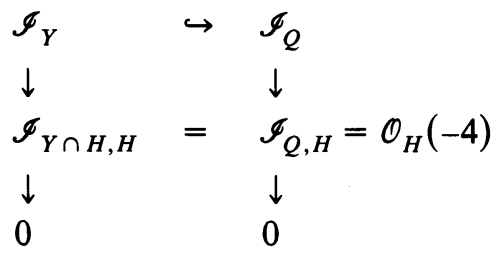

yields, by restricting on $Q$ and dualizing

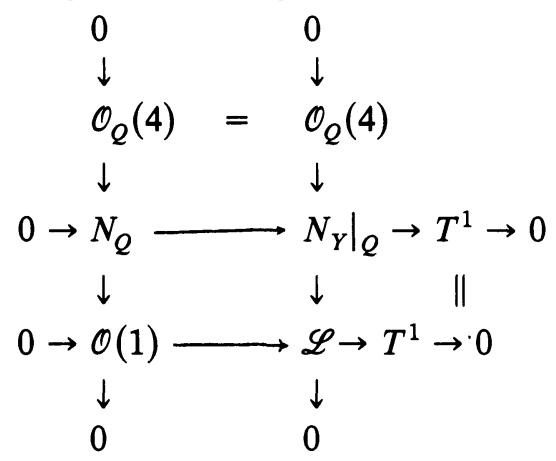


where $\mathscr{L}$ is a line bundle of degree 14 on $Q$. Since $H^{1}(\dot{\mathscr{L}})=H^{1}\left(\mathcal{O}_{Q}(4)\right)=0$, we have $H^{1}\left(\left.N_{Y}\right|_{Q}\right)=0$. Thus $H^{1}\left(N_{Y}\right)=0$.

Lemma 2.4. If $Y=D \cup Q$ is in $V$ and $D$ is general, then $Y$ is linearly normal, $\mu_{0}(Y): H^{0}\left(\mathcal{O}_{Y}(1)\right) \otimes H^{0}\left(\omega_{Y}(-1)\right) \rightarrow H^{0}\left(\omega_{Y}\right)$ is surjective, and $\mathcal{O}_{Y}(2)$ is nonspecial.

Proof. Linear normality of $Y$ follows from [5], and nonspecialness of $\mathcal{O}_{Y}(2)$ follows from the sequence

$$
\left.0 \rightarrow \omega_{Q}(-2) \rightarrow \omega_{Y}(-2) \rightarrow \omega_{Y}(-2)\right|_{D}=\omega_{D}(-1) \rightarrow 0 .
$$

For surjectivity of $\mu_{0}$, consider the following diagram, where $W=H^{0}\left(\mathcal{O}_{\mathrm{P}^{3}}(1)\right)$;

$$
\begin{gathered}
0 \rightarrow W=H^{0}\left(\omega_{0}(-1)\right) \otimes W \rightarrow H^{0}\left(\left.\omega_{Y}(-1)\right|_{D}\right) \otimes W=H^{0}\left(\omega_{D}\right) \otimes W \rightarrow H^{0}\left(\omega_{Y}(-1)\right) \otimes W \rightarrow 0 \\
\alpha \downarrow \\
\beta \downarrow \\
\left.0 \longrightarrow H^{0}\left(\omega_{Q}\right) \longrightarrow \omega_{D}(1)\right) \longrightarrow H^{0}\left(\omega_{Y}\right) \longrightarrow
\end{gathered}
$$

By considering $H^{1}$ terms and using duality, we see easily that both rows are exact. Since $\alpha$ is evidently surjective, it suffices to prove $\beta$ surjective, an assertion completely independent of $Q$. For this, we specialize $D$ to $\bar{D}=C \cup R$, where $C$ is a twisted cubic and $R$ is a curve of genus 2, degree 7 meeting $C$ in two points. As above, we have an exact diagram:

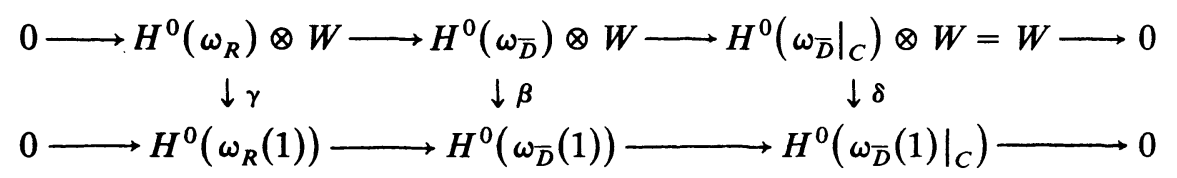

It is easy to see that $\delta$ is bijective, hence it suffices to show $\gamma$ surjective, or equivalently, injective. Suppose $\omega_{1} \otimes H_{1}+\omega_{2} \otimes H_{2} \in \operatorname{ker} \gamma, \omega_{1}, \omega_{2} \in H^{0}\left(\omega_{R}\right)$ independent. Let $A_{i}=\left(\omega_{i}\right)_{0}, B_{i}=\left(H_{i}\right)_{0}$. Then $A_{1}+B_{1}=A_{2}+B_{2}$. Since $A_{1} \cap A_{2}=\varnothing$, it follows that $B_{1}=A_{2}+C, B_{2}=A_{1}+C$ for some divisor $C$ of degree 5. Then $H_{1} \cap H_{2}$ is a 5-secant line of $R$. However, a general $R$ has no 5-secant line: to see this, specialize $R$ to the union of a curve of type $(2,3)$ on a quadric plus a 1-secant conic.

Now, as we shall see below, a general curve in $V$ has maximal rank. On the other hand, there is an obvious way to produce nonmaximal rank curves in $V$ : namely take $D$ in $H_{10,3}$ and a line $L$ on a plane $H$, disjoint from $D$, such that $D \cup L$ has maximal rank. Then $D \cup L$ is contained in a pencil of quintics, and if $Q$ is the residual intersection of one of these with $H$, then $Y=Q \cup D$ has nonmaximal rank, i.e. $h^{0}\left(\mathscr{I}_{Y}(5)\right)=1$ instead of 0 . Let $V_{0} \subset V$ denote the closure of the locus of curves obtained as above. Clearly $V_{0}$ is irreducible. The fact that $V_{0}$ is nonempty is a special case of the next lemma. 
Lemma 2.5. Let $D$ be a general curve in $H_{10,3}$ and $\left\{Q_{t} \mid t \in \mathbf{P}^{1}\right\}$ a general pencil of quartics through $D \cap H$. Then

(i) each $Q_{t}$ is smooth except for finitely many $t$ 's, for which $Q_{t}$ has one ordinary node away from $D \cap H$;

(ii) there exists distinct points $t_{1}, t_{2}, t_{3} \in \mathbf{P}^{1}$ such that for $t \notin\left\{t_{1}, t_{2}, t_{3}\right\}, Y_{t}$ has maximal rank while for $t \in\left\{t_{1}, t_{2}, t_{3}\right\}, Y_{t}$ is in $V_{0}$.

Proof. (i) is general nonsense which follows from the fact that $D \cap H$ is general. For (ii), we specialize $D$ to $\bar{D}$ as in Lemma 2.2. Then $Q_{t}$ meets $H_{2}$ residually in a point $P_{t}$ which moves in a $g_{1}^{1}$ on the line $H \cap H_{2}$. On the other hand, $H_{2}$ meets $L \cup C_{4} \cup C_{5} \cup C_{6}$ residually in five points which lie on a unique conic $C$, and there will be $t_{1}, t_{2} \in \mathbf{P}^{1}$ such that $P_{t} \in C$ iff $t \in\left\{t_{1}, t_{2}\right\}$. For $t \notin\left\{t_{1}, t_{2}\right\}$, if $F_{5}$ is any quintic containing $Y_{t}$ then $F_{5} \cap H_{2}$ contains $E_{2} \cup C$ plus $P_{t} \notin E_{2} \cup C$, hence $F_{5} \supset H_{2}$, i.e. $F_{5}=H_{2}+F_{4}$. Now over on $H_{4}$, the two residual intersections of $Q_{t}$ with $H_{4}$ give a $g_{2}^{1}$ on $H \cap H_{4}$, while the pencil of conics through $H_{4} \cap\left(C_{5} \cup C_{6}\right)$ give another such $g_{2}^{1}$. These two $g_{2}^{1}$ 's meet in a unique point in $\operatorname{Sym}^{2}\left(H \cap H_{4}\right) \simeq \mathbf{P}^{2}$, which corresponds to some $t_{3} \in \mathbf{P}^{1}$. Thus for $t \notin\left\{t_{1}, t_{2}, t_{3}\right\}$ the residual intersection of $Q_{t}$ with $H_{4}$ is not contained in any conic through $H_{4} \cap\left(C_{5} \cup C_{6}\right)$, and this easily implies $F_{4} \supset H_{4}$. We can then continue and "peel off" $H$, then $H_{5}$ and finally use the fact that $L \cup C_{6}$ is not contained in a plane to conclude $H^{0}\left(\mathscr{I}_{Y_{t}}(5)\right)=0$, so that $Y_{t}$ has maximal rank.

It remains to show that $Y_{t_{i}} \in V_{0}, i=1,2,3$. This will follow by looking at our situation another way. The fact that $H^{0}\left(\mathscr{I}_{\bar{D} \cup Q_{t}}(5)\right)=0$ for general $t$ implies that $H^{0}\left(\mathscr{I}_{\bar{D}}(5)\right)=8$, and it is easy to see that $H^{0}\left(\mathscr{I}_{\bar{D}}(4)\right)=0$. Hence we have an injection $\mathbf{P}\left(H^{0}\left(\mathscr{I}_{\bar{D}}(5)\right)\right) \rightarrow \mathbf{P}\left(H^{0}\left(\mathscr{I}_{\bar{D} \cap H, H}(5)\right)\right)=: \mathbf{P}^{10}$ whose image we call $\mathbf{P}^{7}$. On the other hand, we have an embedding $\mathbf{P}^{4} \times H^{\vee} \rightarrow \mathbf{P}^{10}$ given by multiplication, where $\mathbf{P}^{4}=\mathbf{P}\left(H^{0}\left(\mathscr{I}_{\bar{D} \cap H, H}(4)\right)\right), \quad H^{\vee}=\mathbf{P}\left(H^{0}\left(\mathcal{O}_{H}(1)\right)\right)$, whose image is a projection of a Segre variety. Let $I=\mathbf{P}^{4} \times H^{\vee} \cap \mathbf{P}^{7}$, and let $J$ be the image of $I$ in $\mathbf{P}^{4}$; then the expected codimension of $J$ in $\mathbf{P}^{4}$ is 1 and the expected degree of $J$ is 3 (namely this degree is the coefficient of $H_{1} H_{2}^{2}$ in $\left(H_{1}+H_{2}\right)^{3}$, where $H_{1}, H_{2}$ are hyperplane classes on $\mathrm{P}^{4}$ and $H^{\vee}$, respectively). On the other hand, our pencil $\left\{Q_{t}\right\}$ above corresponds to a line $\mathbf{P}^{1} \subset \mathbf{P}^{4}$, and the argument above shows that $\mathbf{P}^{1} \cap J=\left\{t_{1}, t_{2}, t_{3}\right\}$. From this it follows that $J$ is in fact a cubic hypersurface in $\mathbf{P}^{4}$, and is smooth near $Q_{t_{i}}, i=1,2,3$. Moreover, the fact that each $Y_{t_{i}}$ is contained in a unique quintic $F_{i}$ (easily checked) means that the map $I \rightarrow J$ is an isomorphism locally over $Q_{t_{i}}$, with, say, $\left(Q_{t_{i}}, L_{i}\right)$ projecting to $Q_{t_{i}}$. But now, imposing a line on a quintic is given locally by six equations, so for any small perturbation $L$ of $L_{i}$ there will exist $F$ near $F_{i}$ containing $L$, which will then yield a point on the unique component of $I$ containing $\left(Q_{t_{i}}, L_{i}\right)$. Thus the unique component of $I$ containing $\left(Q_{t_{i}}, L_{i}\right)$ 
is 3-dimensional and dominates $H^{\vee}$. But clearly there is at most one 3dimensional component of $I$ dominating $H^{\vee}$ : namely that corresponding to $V_{0}$. In particular, it follows that $Y_{t_{i}} \in V_{0}, i=1,2,3$.

Lemma 2.6. If $Y=Q \cup D$ is a general curve in $V_{0}$ and $F$ is the unique quintic containing $Y$, then $F$ has at most finitely many ordinary double points.

Proof. Specialize $D$ to

$$
\bar{D}=E \cup C,
$$

where $E$ is a cubic in a plane $H^{\prime}$ and $C$ is a curve of degree 7, genus 2 meeting $E$ in one point. It is easy to see [4] that a general $C$ has maximal rank, i.e. $H^{1}\left(\mathscr{I}_{C}(3)\right)=0$, hence a general quartic containing $C$ is smooth. Since $C$ meets $H^{\prime}$ residually in six general points, it is easy to see that the quintics containing $\bar{D}$ are precisely those of the form $H^{\prime}+G$, where $G$ is a quartic surface containing $\bar{D}$. Note that any quartic $Q \subset H$ through $C \cap H$ lifts to a unique quartic surface containing $C$. We can specialize a general pencil $\left\{Y_{t}=Q_{t} \cup D\right\}$ to a pencil $\left\{\bar{Y}_{t}=\bar{Q}_{t} \cup \bar{D}\right\}$, where $\bar{Q}_{t}=G_{t} \cap H$ with $\left\{G_{t}\right\}$ a general pencil of quartics containing $C$ plus three general points on $H \cap H^{\prime}$; note that a general $G_{t}$ is smooth and meets $H^{\prime}$ transversely. Now each of the three quintics $F_{i}, i=1,2,3$, containing the nonmaximal rank curves in our general pencil $\left\{Y_{t}\right\}$ will specialize to some $G_{t}$ and this $G_{t}$ will be a general one in our pencil, because a general quartic $Q \subset H$ through the ten points is a component of some $Y_{t} \in V_{0}$ as follows from the degeneration of Lemma 2.2. Since $F_{i}$ is clearly irreducible and a deformation of a surface with global normal crossings, it follows that $F_{i}$ has at most finitely many ordinary double points, as claimed.

Now for a general pencil $\left\{Y_{t}=Q_{t} \cup D\right\}$ as above, let $\mathscr{Y} \subset \mathbf{P}^{1} \times \mathbf{P}^{3}$ be its total space, and $\pi$ the projection to $\mathbf{P}^{1}$.

Lemma 2.7. For the pencil above, we have

(i) $\pi_{*}\left(\omega_{g / \mathbf{P}^{1}}(0,-1)\right)=\mathcal{O}_{\mathbf{P}^{1}}(1) \oplus 3 \mathcal{O}_{\mathbf{P}^{1}}$;

(ii) $R^{1} \pi_{*}\left(\mathscr{I}_{\mathscr{Q}}(0,2)\right)=40$.

Proof. (i) For each $Y_{t}=Q_{t} \cup D$, we have an exact sequence

$$
0 \rightarrow H^{0}\left(\omega_{Q_{t}}(-1)\right) \rightarrow H^{0}\left(\omega_{Y_{t}}(-1)\right) \rightarrow H^{0}\left(\omega_{D}\right) \rightarrow 0
$$

which globalizes to

$$
0 \rightarrow \pi_{*}\left(\omega_{\mathscr{Q} / \mathbf{P}^{1}}(0,-1)\right) \rightarrow \pi_{*}\left(\omega_{\mathscr{Y} / \mathbf{P}^{1}}(0,-1)\right) \rightarrow H^{0}\left(\omega_{D}\right) \otimes \mathcal{O}_{\mathbf{P}^{1}} \rightarrow 0
$$

where $\mathscr{Q} \subset \mathbf{P}^{1} \times H$ is the total space of the pencil $\left\{Q_{t}\right\}$. Since $\mathscr{Q}$ is just a divisor of type $(1,4)$, it follows easily by adjunction that $\pi_{*} \omega_{\mathscr{Q} / \mathbf{P}^{1}}(0,-1)=\mathcal{O}(1)$, which implies (i). For (ii), use the exact sequence

$$
0 \rightarrow \mathscr{I}_{D}(1) \rightarrow \mathscr{I}_{Y_{t}}(2) \rightarrow \mathcal{O}_{H}(-2) \rightarrow 0
$$

to get a canonical isomorphism $H^{1}\left(\mathscr{I}_{Y_{t}}(2)\right) \simeq H^{1}\left(\mathscr{I}_{D}(1)\right)$, a fixed 4dimensional vector space. 
Now using the results of $\S 1$, we can associate to $\mathscr{Y} \subset \mathbf{P}^{1} \times \mathbf{P}^{3}$ a vector bundle $\mathscr{E}$ on $\mathbf{P}^{1} \times \mathbf{P}^{3}$ plus an exact sequence

$$
0 \rightarrow 3 \mathcal{O}(1,0) \oplus \mathcal{O} \rightarrow \mathscr{E}(0,2) \rightarrow \mathscr{I}_{\mathscr{Y}}(1,5) \rightarrow 0
$$

and a monad

$$
0 \rightarrow \mathscr{E} \rightarrow 8 \mathcal{O}(1,0) \oplus \mathcal{O}(0,-1) \stackrel{\Phi}{\rightarrow} 4 \mathcal{O}(1,1) \rightarrow 0 .
$$

Now let

$$
\begin{gathered}
\mathbf{P}_{A}=\mathbf{P}\left(\operatorname{Hom}\left(8 H^{0}\left(\mathcal{O}_{\mathbf{P}^{3}}\right)\right), 4 H^{0}\left(\mathcal{O}_{\mathbf{P}^{3}}(1)\right)\right), \\
\mathbf{P}_{B}=\mathbf{P}\left(\operatorname{Hom}\left(H^{0}\left(\mathcal{O}_{\mathbf{P}^{3}}(-1)\right)\right), 4 H^{0}\left(\mathcal{O}_{\mathbf{P}^{3}}(1)\right)\right) .
\end{gathered}
$$

Then the map $\Phi$ above corresponds to a map $\mathbf{P}^{1} \rightarrow \mathbf{P}_{A} \times \mathbf{P}_{B}$ of bidegree $(0,1)$ whose image we call $P \times L$, where $P \in \mathbf{P}_{A}$ and $L$ is a line in $\mathbf{P}_{B}$. Now let $P^{\prime}$ be a general point in $\mathbf{P}_{A}, L^{\prime}$ a general line in $\mathbf{P}_{B}, \Phi^{\prime}$ a map as above corresponding to $P^{\prime} \times L^{\prime}$, and $\mathscr{E}^{\prime}$ the corresponding sheaf, i.e., $\operatorname{ker} \Phi^{\prime}$. Note that $\Phi^{\prime}$ is surjective because $\Phi$ is, hence $\mathscr{E}^{\prime}$ is locally free.

Lemma 2.8. (i) $h^{1}\left(E_{t}^{\prime}(2)\right)=0$ and $h^{0}\left(E_{t}^{\prime}(2)\right)=4$ for all $t$.

(ii) The dependency locus of the map $\pi^{*} \pi_{*} \mathscr{E}^{\prime}(0,2) \rightarrow \mathscr{E}^{\prime}(0,2)$ is a flat family $\mathscr{Y}^{\prime} \rightarrow \mathbf{P}^{1}$ of Cohen-Macaulay curves with smooth general fiber.

Proof. Recall that for our special family $\mathscr{Y}$ and special bundle $\mathscr{E}$ we have $h^{0}\left(E_{t}(2)\right)=5$ for $t \in\left\{t_{1}, t_{2}, t_{3}\right\}$, and $h^{0}\left(E_{t}(2)\right)=4$ for $t \notin\left\{t_{1}, t_{2}, t_{3}\right\}$. Since $H^{1}\left(N_{Y_{t}}\right)=0$ for all $t$, our family $\left\{Y_{t}\right\}$ is contained in a unique component of the Hilbert scheme, say $\mathscr{H}$, whose general element is, as we have seen (Lemma 2.2), smooth. Now $Y_{t_{i}}$ corresponds to a particular 4-dimensional subspace $W_{i} \subset H^{0}\left(E_{t_{i}}(2)\right)$, and by varying $W_{i}$ within $H^{0}\left(E_{t_{i}}(2)\right)$ we obtain a 4dimensional family of distinct (by the Serre construction) curves in $\mathscr{H}$. This shows that the rational map $\mathbf{P}_{A} \times \mathbf{P}_{B} \rightarrow$ bundles $\} \rightarrow \mathscr{H}$ blows up (in fact, becomes one-to- $\left.\infty^{4}\right)$ at the point corresponding to $E_{t_{i}}$. It follows that the locus of matrices in $\mathbf{P}_{A} \times \mathbf{P}_{B}$ corresponding to bundles with $h^{0}(E(2))>4$ (which is equivalent to $h^{1}(E(2))>0$ ) has codimension $\geqslant 2$ (in fact, $\geqslant 5$ ) near the point corresponding to $E_{t_{i}}$, hence that locus will be missed by a general $P^{\prime} \times L^{\prime}$. This proves (i). For (ii), note that by the exact sequence

$$
0 \rightarrow W_{i} \otimes \mathcal{O} \rightarrow E_{t_{i}}(2) \rightarrow \mathscr{I}_{Y_{t_{i}}}(5) \rightarrow 0
$$

and the fact that $h^{0}\left(\mathscr{I}_{\mathrm{Y}_{\mathrm{t}}}(5)\right)=1$, it follows that an arbitrary 4-dimensional subspace $W_{i} \subset H^{0}\left(E_{t_{i}}(2)\right)$ has the property that for some 3-dimensional subspace $U \subset W_{i}$, the cokernel $\bar{E}_{t_{i}}(2)$ of the map $U \otimes \mathcal{O} \rightarrow E_{t_{i}}(2)$ is a reflexive sheaf of rank 2; and, of course, the same is true for any $t \notin\left\{t_{1}, t_{2}, t_{3}\right\}$. It follows that for our general bundle $\mathscr{E}^{\prime}$ the same is true for $E_{t}^{\prime}$ for all $t$. Now 
the dependency locus of $H^{0}\left(E_{t}^{\prime}(2)\right) \otimes \mathcal{O} \rightarrow E_{t}^{\prime}(2)$ coincides with the 0-locus of some nonzero section of $\bar{E}_{t}(2)$. But the fact that $H^{0}\left(E_{t}(1)\right)=0$ clearly implies $H^{0}\left(\bar{E}_{t}(1)\right)=0$, hence no section of $\bar{E}_{t}(2)$ can vanish on a surface. This completes the proof of (ii).

Lemma 2.9. For general $Y=Q \cup D$ in $V_{0}$, the unique quintic $F$ containing $Y$ is smooth along $Y$, and $\left|\mathcal{O}_{F}(Y)\right|$ is base-point-free and of dimension 4, and coincides with the closure of the set of curves corresponding to the same bundle as $Y$.

Proof. We already know $F$ has at most finitely many double points. We have an exact sequence

$$
0 \rightarrow W \otimes \mathcal{O} \rightarrow E(2) \rightarrow \mathscr{I}_{Y}(5) \rightarrow 0 .
$$

Now deforming the subspace $W \subset H^{0}(E(2))$ yields deformations of $Y$ in $F$. Recall that $W=H^{0}\left(\omega_{Y}(-1)^{\vee}\right.$, so infinitesimal deformations of $W$ in $H^{0}(E(2))$ are in 1-1 correspondence with sections of $\omega(-1)$. As $\omega(-1)$ has no base points, it follows that by moving $W, Y$ moves in $F$ without base points. This easily implies that $F$ is smooth along the smooth part of $Y$; on the other hand, $F$ is smooth at $\operatorname{sing}(Y)=D \cap Q$ because $F \cap H=Q \cup L$ is smooth there. Thus $F$ is smooth along $Y$, and in particular $Y$ is a Cartier divisor on $F$. Now the adjunction formula gives $\left.\mathcal{O}_{F}(Y)\right|_{Y}=\mathcal{O}_{Y}(Y)=\omega_{Y}(-1)$, and the sequence

$$
0 \rightarrow \mathcal{O}_{F} \rightarrow \mathcal{O}_{F}(Y) \rightarrow \mathcal{O}_{Y}(Y) \rightarrow 0
$$

and $H^{1}\left(\mathcal{O}_{F}\right)=0$ give $h^{0}\left(\mathcal{O}_{F}(Y)\right)=5$. Since deformations of $W$ give us (distinct) elements of $\left|\mathcal{O}_{F}(Y)\right|$, it follows for dimension reasons that the closure of the set of curves coming from deformations of $W$ coincides with $\left|\mathcal{O}_{F}(Y)\right|$, as claimed.

Lemma 2.10. Except for a codimension-2 locus, the curves in $\left|\mathcal{O}_{F}(Y)\right|$ have at most ordinary nodes and ordinary tacnodes as singularities.

Proof. Recall that $F \cap H=Q \cup L$. It suffices to prove that our assertion is true in a neighborhood of some pencil in $\left|\mathcal{O}_{F}(Y)\right|$, e.g. the pencil $\left\{Q_{t} \cup D\right\}$ obtained by varying $H$ through $L$. Now specialize $D$ to $\bar{D}=E \cup C$ as in Lemma 2.6. Then $F$ specializes to $G+H^{\prime}$ as there, and our pencil $\left\{Q_{t} \cup D\right\}$ specializes to the following:

(a) the pencil $\left\{\left(H_{t} \cap G\right) \cup D \mid H_{t} \supset L\right\}$, plus

(b) $\left\{D \cup\left(H^{\prime} \cap G\right) \cup B_{t}\right\}$, where $B_{t}$ is some pencil of cubics on $H^{\prime}$ through $E^{\prime} \cap L$.

It is easy to see that, except for some codimension-2 locus, the only singularities which occur in a neighborhood of the above families are as follows:

(i) normal crossings;

(ii) ordinary tacnodes (which occur when $H_{t}$ becomes tangent to $D$ ); 
(iii) normal crossings plus an embedded point, occurring at some of the intersections of $B_{t}$ with $G$.

Now the singularities of type (iii) cannot survive on our general $F$, because such curves cannot be the limit of a reduced curve on a surface with Q-factorial singularities. This proves the lemma.

We can now state the qualitative result which the above discussion has been leading to.

Theorem 2.11. For a general bundle $\mathscr{E}^{\prime}$ on $\mathbf{P}^{1} \times \mathbf{P}^{3}$ as above, the dependency locus of $\pi^{*} \pi_{*} \mathscr{E}^{\prime}(0,2) \rightarrow \mathscr{E}^{\prime}(0,2)$ is a family $\left\{Y_{t}^{\prime}\right\}$ of stable, automorphismfree curves in $\mathscr{M}_{15} \cup \Delta_{0}$.

Proof. As $\mathscr{E}^{\prime}$ specializes to our special bundle $\mathscr{E}$, the family $\left\{Y_{t}^{\prime}\right\}$ specializes to $\left\{Y_{t}\right\}$ plùs some 1-dimensional family in $\left|\mathcal{O}_{F_{i}}\left(Y_{t_{i}}\right)\right|$ for $i=1,2,3$. Since we can avoid any codimension-2 locus by deforming $\mathscr{E}^{\prime}$, it follows that our curves $Y_{t}^{\prime}$ have at most ordinary nodes and ordinary tacnodes, and are smooth for generic $t$.

Claim. For each $t$ we have

(i) $Y_{t}^{\prime}$ is linearly normal and $\mu_{0}\left(Y_{t}^{\prime}\right)$ is surjective.

(ii) $H^{1}\left(N_{Y_{t}^{\prime}}\right)=0$; equivalently, $h^{0}\left(N_{Y_{t}^{\prime}}\right)=56$.

(i) follows from the fact (cf. Proposition 1.3) that linear normality and surjectivity of $\mu_{0}$ can be interpreted in terms of the corresponding bundle $E_{t}^{\prime}$, and that the corresponding properties are true for $E_{t}$ for all $t$. For (ii), we use a similar argument: namely note that the functorial correspondence between a maximal rank curve $Y$ and its bundle extends to a correspondence between their deformation functors, and in particular we have $H^{0}\left(N_{Y_{t}}\right) \simeq$, $H^{1}\left(E_{t}^{\prime} \otimes E_{t}^{\prime \vee}\right)$. Since $H^{i}\left(E_{t}^{\prime} \otimes E_{t}^{\prime \vee}\right)=0$ for $i \geqslant 2$, as we can check easily from the monad, and $\chi\left(E_{t}^{\prime} \otimes E_{t}^{\prime \vee}\right)=-55$ by Riemann-Roch, it suffices to prove $H^{0}\left(E_{t}^{\prime} \otimes E_{t}^{\prime \vee}\right)=$ scalar endomorphisms $\}$, i.e. $E_{t}^{\prime}$ is simple. Let $\phi \in$ $H^{0}\left(E_{t}^{\prime} \otimes E_{t}^{\prime \vee}\right)$. We have a diagram:

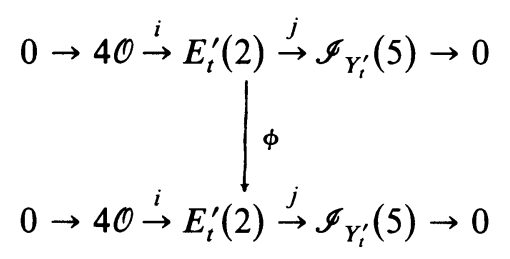

Since $H^{0}\left(\mathscr{I}_{Y^{\prime}}(5)\right)=0$, the map $j \circ \phi \circ i$ must vanish, hence $\phi$ induces $\bar{\phi}: \mathscr{I}_{Y_{t}^{\prime}}(5) \rightarrow \mathscr{I}_{Y_{t}^{\prime}}(5)$, which must be multiplication by some scalar $\alpha$. Then $\phi-\alpha \cdot$ id maps $E_{t}^{\prime}(2)$ to $4 \mathcal{O}$, hence vanishes. This proves the claim. 
Thus the component $\mathscr{H}$ of the Hilbert scheme is smooth in a neighborhood of our family $\left\{Y_{t}^{\prime}\right\}$. Now the surjectivity of $\mu_{0}\left(Y_{t}^{\prime}\right)$ has the following interpretation: Let $B$ be the base of the versal deformation of $Y_{t}^{\prime}$; then the map $\mathscr{H} \rightarrow B$ has image a smooth divisor $D$ in $B$ and its fibers are just the orbits of PGL $_{3}$. Now suppose $Y_{t}^{\prime}$ has a tacnode. It is easy to see that the locus $T$ of tacnodal curves in $B$ has codimension 3 , hence $T \cap D$ has codimension $\leqslant 2$ in $D$. Hence the locus of tacnodal curves in $\mathscr{H}$ has codimension $\geqslant 2$ near $Y_{t}^{\prime}$, so it can be avoided by deforming $\mathscr{E}^{\prime}$.

Thus for all $t, Y_{t}^{\prime}$ is a nodal curve. Since $Y_{t}^{\prime}$ is linearly normal, the only way $Y_{t}^{\prime}$ could fail to be stable is if $Y_{t}^{\prime}=A \cup L$, where $L$ is a 2-secant line of $A$; but this contradicts the fact that $\omega_{Y_{t}^{\prime}}(-1)$ has no base points. Thus all $Y_{t}^{\prime}$ are stable. By linear normality again, no $Y_{t}^{\prime}$ can be in $\Delta_{i}$ for $i>0$. The fact that the $Y_{t}^{\prime}$ are automorphism-free follows from the fact that the locus of curves with automorphisms has codimension $\geqslant 3$ in $\overline{\mathscr{M}}_{15}$ (away from $\bigcup_{i>0} \Delta_{i}$ ).

\section{Computations; conclusion}

We begin with some generalities about families of curves. Let $\pi: \mathscr{Y} \rightarrow B$ be a family of stable curves over a smooth curve $B$. Note that $\mathscr{Y}$ is a locally complete intersection surface, hence has virtual cotangent bundle $\Omega_{\mathscr{Y}}^{1}$ (defined by means of some embedding of $\mathscr{Y}$ in a smooth variety). We have

$$
\omega_{\mathscr{Y} / B}=c_{1}\left(\Omega_{\mathscr{Y}}^{1}\right) \otimes \omega_{B}^{-1} .
$$

Recall that $\kappa=\left[\omega_{\mathscr{O} / B}\right]^{2}$, hence

$$
\kappa=c_{1}^{2}\left(\Omega_{\mathscr{Y}}^{1}\right)+8(1-h)(g-1),
$$

where $h$ is the genus of $B$ and $g$ is the genus of a fiber. Also, the locus of singular points of fibers of $\pi$ is given by the 0-locus of $d \pi: \pi^{*} \Omega_{B} \rightarrow \Omega_{\mathscr{Y}}^{1}$, hence the (weighted) number $\delta$ of singular fibers is given by $c_{2}\left(\Omega_{\mathscr{y}}^{1} \otimes \pi^{*} \Omega_{B}^{\vee}\right)$, i.e.

$$
\delta=c_{2}\left(\Omega_{\mathscr{y}}^{1}\right)+4(1-h)(g-1)
$$

Finally, recall Mumford's formula [12]

$$
\kappa=12 \lambda-\delta,
$$

where $\lambda=c_{1}\left(\pi_{*} \omega_{\mathscr{y} / B}\right)$.

Next we recall a formula of Eisenbud and Harris [6].

Theorem (Eisenbud-Harris). Let $g+1=(r+1) s$ and let $D \subset \overline{\mathscr{M}}_{g}$ be a divisor contained in the closure of $D_{s}^{r}=\left\{C \in \mathscr{M}_{g} \mid C\right.$ has a $g_{d}^{r}$, where $d=g-s$ $+r\}$. Then the class of $D$ in $\operatorname{Pic}\left(\overline{\mathscr{M}}_{g}\right)$ is given by $[D]=a \lambda-b \delta_{0}-\sum c_{i} \delta_{i}$, where $a / b=6+12 /(g+1)$ and $a>0$. 
Proof. This result is proved in [6], under the additional hypothesis that $D$ coincides with the codimension-1 part of $\bar{D}_{s}^{r}$, but the proof given there actually proves the above statement (as well as determining $a / c_{i}$, which we do not care about).

Next, we recall a formula of Harris and Tu [10].

Theorem (Harris-Tu). Let $M$ be a smooth variety of dimension 4 and $f: A \rightarrow B$ a homomorphism between vector bundles of rank $a$ and $a+1$, respectively. Suppose the locus $Z \subset M$, where $f$ has rank $<a$, is a locally complete intersection surface. Then the virtual Chern numbers of $Z$ are given by

$$
\begin{aligned}
c_{1}^{2}(Z)= & {\left[\left(c_{1}(M)-c_{1}\right)^{2}-2\left(c_{1}(M)-c_{1}\right) c_{1}+c_{1}^{2}\right] c_{2}, } \\
c_{2}(Z)=[ & \left(c_{2}(M)-c_{1}(M) c_{1}+c_{2}(A)-c_{2}(B)-c_{1}(A) c_{1}(B)\right. \\
& \left.+c_{1}^{2}(B)+\left(c_{1}(M)+2 c_{1}\right) c_{1}+c_{2}-2\left(c_{1}^{2}-c_{2}\right)\right] c_{2},
\end{aligned}
$$

where $c_{i}=c_{i}(F-E)$.

Proof. This is a special case of a formula given in [10], under the additional hypothesis that $Z$ is smooth. However, the proof given there applies in the above generality.

We shall apply the Harris-Tu formula to the map $\pi^{*} \pi_{*} \mathscr{E}^{\prime}(0,2) \rightarrow \mathscr{E}^{\prime}(0,2)$ of Theorem 2.11. By Example 1.6 we have

$$
c_{1}(\mathscr{E}(0,2))=4 H_{1}+5 H_{2}, \quad c_{2}(\mathscr{E}(0,2))=16 H_{1} H_{2}+14 H_{2}^{2} .
$$

Now of course $c_{i}(\mathscr{E}(0,2))=c_{i}\left(\mathscr{E}^{\prime}(0,2)\right)$, and the monad

$$
0 \rightarrow \mathscr{E}^{\prime}(0,2) \rightarrow 8 \mathcal{O}(1,2) \oplus \mathcal{O}(0,1) \rightarrow 4 \mathcal{O}(1,2) \rightarrow 0
$$

plus $R^{1} \pi_{*} \mathscr{E}^{\prime}(0,2)=0$ yield $c_{1}\left(\pi_{*} \mathscr{E}^{\prime}(0,2)\right)=0$. Thus in the situation of the Harris-Tu formula we have $c_{1}=4 H_{1}+5 H_{2}, c_{2}=16 H_{1} H_{2}+14 H_{2}^{2}$. Plugging this in yields $c_{1}^{2}(Z)=1584, c_{2}(Z)=2064$, hence $\kappa=1696, \delta=2120, \lambda=$ 318 , so finally the Eisenbud-Harris formula plus Mumford's formula $K_{\mathscr{M}_{\mathrm{g}}}=$ $13 \lambda-2 \delta-\delta_{1}$ yield:

Corollary 3.1. If $\mathscr{Y}^{\prime} \rightarrow \mathbf{P}^{1}$ is as in Theorem 2.11, and $f=\mathbf{P}^{1} \rightarrow \overline{\mathscr{M}}_{15}$ is the associated map, then $\operatorname{deg} f^{*} D>0$, and $\operatorname{deg} f^{*} K_{\mathscr{M}_{15}}=-106<0$.

As $f\left(\mathbf{P}^{1}\right)$ moves freely on $D$, we have $f\left(\mathbf{P}^{1}\right) \cdot B \geqslant 0$ for any prime divisor $B \neq D$, hence we conclude

Corollary 3.2. $f\left(\mathbf{P}^{1}\right)$ is a numerically effective curve in $\mathscr{M}_{15}$.

Corollaries 3.1 and 3.2 yield immediately

Theorem 3.3. The Kodaira dimension of $\mathscr{M}_{15}$ is $-\infty$.

\section{References}

[1] E. Arbarello \& M. Cornalba, Su una congettura di Petri, Comment. Math. Helv. 56 (1981) 1-38.

[2] E. Arbarello \& E. Sernesi, The equation of a plane curve, Duke Math. J. 46 (1979) 469-485. 
[3] W. Barth \& K. Hulek, Monads and moduli of vector bundles, Manuscripta Math. 25 (1978) 323-347.

[4] M. Chang, Stable rank-2 reflexive sheaves on $\mathbf{P}^{3}$ with small $c_{2}$ and applications, Trans. Amer. Math. Soc. 284 (1984) 57-89.

[5] M. Chang \& Z. Ran, Unirationality of the moduli spaces of curves of genus 11, 13 (and 12), Invent. Math. 76 (1984) 41-54.

[6] D. Eisenbud \& J. Harris, On the Kodaira dimension of the moduli space of curves of genus $\geqslant 23$, preprint.

[7] W. Fulton, Intersection theory, Springer, Berlin, 1984.

[8] J. Harris, On the Kodaira dimension of the moduli space of curves, II. The even-genus case, Invent. Math. 75 (1984).

[9] J. Harris \& D. Mumford, On the Kodaira dimension of the moduli space of curves, Invent. Math. 67 (1982) 23-97.

[10] J. Harris \& L. Tu, Chern numbers of kernel and cokernel bundles, Invent. Math. 75 (1984) 467-475.

[11] R. Hartshorne, Stable vector bundles of rank 2 on $\mathbf{P}^{3}$, Math. Ann. 238 (1978) 229-280.

[12] D. Mumford, Stability of projective varieties, Enseign. Math. 23 (1977) 39-110.

[13] E. Sernesi, L' unirazionalità della varietà dei moduli delle curvi di genere dodici, Ann. Scuola Norm. Sup. Pisa Cl. Sci. (4) 8 (1981) 405-439.

[14] _ On the existence of certain families of curves, Invent. Math. 75 (1984) 25-57.

\author{
UNIVERSity OF SOUTH CAROlina \\ UNIVERSITY OF CALIFORNIA, RIVERSIDE
}

